\title{
Macular vessel density versus ganglion cell complex thickness for detection of early primary open-angle glaucoma
}

\author{
Yiwei Wang ${ }^{1,2}$, Chen Xin ${ }^{1,2}$, Meng Li ${ }^{1,2}$, David L. Swain ${ }^{3,4}$ (D) Kai Cao ${ }^{1,2}$, Huaizhou Wang ${ }^{2,5}$ and Ningli Wang ${ }^{1,2^{*}}$ (D)
}

\begin{abstract}
Background: To evaluate the macular vessel density (VD) and ganglion cell complex (GCC) thickness in preperimetric (PPG) and early perimetric primary open-angle glaucoma (PG) eyes, and to compare the diagnostic ability of the two measurements to discriminate PPG and early PG eyes from healthy eyes.

Methods: Seventy-nine eyes in 72 subjects (31 normal, 26 PPG, and 22 early PG eyes) were included in the consecutive case series. Macular VD and GCC thickness were acquired simultaneously using the $6 \times 6 \mathrm{~mm}^{2}$ highdensity AngioRetina scanning mode. Diagnostic abilities were assessed using the area under the receiver operating characteristic curve (AUROC).

Results: Compared to healthy eyes, whole image VD (wiVD) and GCC thickness were significantly lower in PPG and early PG eyes (all $P<0.025$ ). The percent reduction of wiVD was lower than that of GCC thickness in early PG eyes $(P<0.05)$, while they were similar in PPG eyes $(P>0.05)$. Regionally, greater VD attenuation and GCC thinning were identified in the perifovea than in the parafovea in both groups (all $P<0.05$ ). Moreover, the percent reduction of VD was less than that of GCC thickness in the perifoveal region in PPG eyes $(P<0.05)$. The AUROCs for wiVD and GCC thickness were 0.824 and 0.881 , respectively, in PPG eyes $(P>0.05)$, and 0.918 and 0.977 , respectively, in early PG eyes $(P>0.05)$.

Conclusions: Macular VD and GCC thickness significantly decreased in PPG and early PG eyes. The perifoveal region appeared to be more vulnerable to macular VD attenuation and GCC thinning in early glaucoma. Our results showed that macular VD measurements may be helpful for detecting and understanding early glaucomatous damage.
\end{abstract}

Keywords: Primary open-angle glaucoma, Pre-perimetric glaucoma, Macular vessel density, Ganglion cell complex, Optical coherence tomography angiography

\section{Background}

Primary open-angle glaucoma (POAG) is a leading cause of irreversible blindness worldwide [1], and it is characterized by progressive degeneration of retinal ganglion cells (RGCs) and their axons [2]. Many factors are involved in the development of POAG, including vascular insufficiency [3-6]. Since RGCs are nourished by the

\footnotetext{
* Correspondence: wningli@vip.163.com

${ }^{1}$ Beijing Institute of Ophthalmology, Beijing Tongren Eye Centre, Beijing

Tongren Hospital, Capital Medical University, 1 Dongjiaominxiang Street,

Dongcheng District, Beijing 100730, China

${ }^{2}$ Beijing Ophthalmology \& Visual Sciences Key Laboratory, 1

Dongjiaominxiang Street, Dongcheng District, Beijing 100730, China

Full list of author information is available at the end of the article
}

superficial retinal capillary plexus, it is presumed that decreased superficial retinal microcirculation in the macular region may occur in the course of glaucoma.

Optical coherence tomography angiography (OCTA) is a noninvasive imaging technique that provides reproducible measurements of vasculature in defined segments by separating static signals from motion signals $[7,8]$. Recent studies have shown decreased superficial retinal microcirculation in the macular region in eyes with POAG [9-15]. More interestingly, Yarmohammadi et al. [16] reported that macular vessel density (VD) attenuated in the perimetrically intact hemiretina of glaucoma eyes with a single-hemifield defect, suggesting that 
significant microvasculature alterations in the macular region may precede detectable visual field (VF) defects.

Early glaucomatous damage has been shown to involve the macula $[17,18]$. Studies have reported that 25 to $50 \%$ of RGCs were lost prior to detectable VF impairment $[19,20]$. Therefore, macular measurements may be potential indicators for early detection of glaucoma. Structural parameters in the macular region such as the ganglion cell complex (GCC) or ganglion cell-inner plexiform layer have been found to be thinner in eyes with pre-perimetric glaucoma (PPG) than in healthy eyes [21-25]. However, only a few studies have focused on macular microcirculation changes in early glaucoma [26-30]. Among these studies, fewer have focused on PPG [28-30]. The current study aimed to evaluate macular VD and GCC thickness in eyes with PPG and early perimetric POAG (PG), and to compare the diagnostic abilities of the two parameters in discriminating PPG or early PG eyes from healthy eyes.

\section{Methods}

This observational study was conducted in Beijing Tongren Hospital between January 2018 and May 2018. The study followed the tenets of the Declaration of Helsinki and was approved by the Institutional Review Board of Beijing Tongren Hospital. Informed consent was obtained from all subjects.

Overall inclusion criteria were an age of $\geq 18$ years, open angles under gonioscopy, a best-corrected visual acuity of 0.5 or better, and a spherical equivalent within +3 to -6 diopters. The exclusion criteria were a prior intraocular surgery except for uncomplicated cataract or glaucoma surgery; non-glaucomatous optic neuropathy and retinopathy, uveitis, or ocular trauma; diagnosis of Parkinson's disease, Alzheimer's disease, or history of stroke; any other known disease that may cause optic neuropathy, retinopathy or VF loss; an unreliable VF; and poor-quality OCTA scans as described later.

Healthy controls were defined as individuals who had an intraocular pressure (IOP) of $<21 \mathrm{mmHg}$ without history of elevated IOP, normal-appearing optic nerve head, intact neuroretinal rim and retinal nerve fiber layer (RNFL), and reliable normal VF. A normal VF was defined as a pattern standard deviation (PSD) within the 95\% confidence limit, and a glaucoma hemifield test result within the normal limits by reliable VF test [31]. A reliable VF test was defined as a VF with a false-positive error of $<15 \%$, a false-negative error of $<15 \%$, and a fixation loss of $<33 \%$. PPG in this study was defined based on the presence of a normal anterior segment on a slitlamp examination, a gonioscopically open angle and glaucomatous optic neuropathy (i.e., optic nerve rim defect (notching or localized thinning), optic disc hemorrhage, and RNFL defect) in the absence of glaucomatous VF abnormalities [7, 30]. Glaucomatous VF abnormalities were defined as follows: (1) PSD outside normal limits $(P<0.05)$ and glaucoma hemifield test outside normal limits; (2) the presence of at least 3 adjacent points within the same hemifield on the pattern deviation probability plot at $P<0.05$ with at least 1 point at $P<0.01$. Early PG eyes showed glaucomatous optic neuropathy, open angles under gonioscopy, and typical glaucomatous VF defects corresponding to glaucomatous optic neuropathy. Early glaucoma was defined as 24-2 MD > $-6 \mathrm{~dB}$.

All participants underwent comprehensive ophthalmic examinations, including assessment of best-corrected visual acuity, slit-lamp biomicroscopy, IOP assessment by Goldmann applanation tonometry, gonioscopy, fundus examination, stereoscopic visualization and photography (Kowa, Japan) of optic nerve head, central corneal thickness (CCT) assessment with the Casia SS-1000 OCT (Tomey, Japan), axial length assessment with the Lenstar Optical Biometer (Haag-Streit, USA), perimetry using the Zeiss Humphrey Field Analyzer (Carl Zeiss Meditec Inc., Dublin, California, USA) with the Swedish interactive threshold algorithm standard 24-2 program, and peripapillary RNFL (pRNFL) thickness assessment with the RTVue XR Avanti with AngioVue system (Optovue Inc., Fremont, CA, USA). Systemic blood pressure (BP) was measured with an Omron automatic BP instrument in an upright sitting position. Mean arterial pressure (MAP) was calculated as follow: $\mathrm{MAP}=$ diastolic BP + 1/3 (systolic BP - diastolic BP). Mean ocular perfusion pressure (MOPP) was calculated based on the following equation: $\mathrm{MOPP}=2 \times(\mathrm{MAP}-\mathrm{IOP}) / 3$.

The macular VD and GCC thickness were acquired simultaneously using the RTVue XR Avanti with AngioVue system by the same operator (W.Y.W). The software version was 2017.1.0.155. The $6 \times 6 \mathrm{~mm}^{2}$ highdensity AngioRetina scanning mode with the ETDRS segmentation algorithm was used in this study. VD was defined as the percentage of the area occupied by vessels in the defined area [32]. We focused on the "superficial vascular complex" (a slab defined by the Angiovue software extending from the internal limiting membrane to the inner plexiform layer $-10 \mathrm{~mm}$ ) in the macular region [15]. The whole image vessel density (wiVD) was measured in the entire $6 \times 6 \mathrm{~mm}^{2}$ image. In addition, the retinal map was segmented using three concentric rings with diameters of 1,3 , and $6 \mathrm{~mm}$. The intermediate (the parafoveal region) and outer (the perifoveal region) rings were divided into 4 sectors of 90 degrees each (para -/peri-nasal, para-/peri-inferior, para-/peri-superior, and para-/peri-temporal sectors). The superiorhemifield and inferior-hemifield VD were obtained by dividing the scan area across the horizontal meridian. GCC thickness was acquired using the $6 \times 6 \mathrm{~mm}^{2}$ high- 
density AngioRetina scanning mode and measured from the inner limiting membrane (ILM) to the posterior boundary of the inner plexiform layer. The corresponding segmented regions listed above were also used for GCC thickness analysis. The percent reduction of macular VD and GCC thickness was calculated using the following equation: (mean value for normal eyes - mean value for glaucomatous eyes) / mean value for normal eyes. The image quality of all OCT angiograms was assessed. Scans with the presence of one of the following issues were excluded from analysis: a signal strength index (SSI) below 50, residual motion artefacts and a weak local signal due to vitreous opacity.

Statistical analyses were performed using SPSS (v20.0; IBM SPSS Inc., Chicago, USA), MedCalc (v15.2.2; MedCalc Software bvba, Ostend, Belgium) and R statistical computing package (v3.6.0; R Foundation for Statistical Computing, Vienna, Austria). Categorical variables were compared using Chi-square test. Age was compared using the Mann-Whitney test. Mixed effects models were used to compare the ocular characteristics. Univariate and multivariate analyses in mixed effects model were used to compare the macular VD and GCC thickness parameters between PPG, early PG, and healthy eyes. Potential confounding factors, such as age, sex, SSI and any ophthalmic characteristics were included in multivariate models if the $P$ value was $<0.05$ in univariate analysis. The clustered Wilcoxon signed rank test using the Rosner-Glynn-Lee method was used to compare the percent reduction of the macular VD and GCC thickness within each individual group. The Spearman correlation and mixed effects models were used to assess the associations between macular VD, GCC thickness and other variables (pRNFL, MD and PSD). The diagnostic ability was evaluated by the area under the receiver operating characteristic curve (AUROC). The DeLong method was used for comparisons between AUROCs. Statistical significance was set at two-tailed $P<0.05$. Bonferroni-corrected significance level was used for multiple comparisons.

\section{Results}

A total of 79 eyes (31 normal, 26 PPG and 22 early PG eyes) from 72 subjects were included for analysis in this study. The demographics and ocular characteristics are summarized in Additional file 1. The age, sex of the subjects, prevalence of a history of hypertension, usage rate of hypotensive drugs, prevalence of diabetes history, usage rate of diabetes drugs, MOPP, CCT, and axial length were not significantly different between the normal and PPG groups, or between the normal and early PG groups (all $P>0.025$ ), except that the normal and PPG groups differed by sex $(P=0.019)$. The difference in the usage rate of hypotensive eye drops between the
PPG and early PG groups was not statistically significant $(P>0.025)$. The average, superior-hemifield, and inferior-hemifield pRNFL in PPG and early PG eyes were significantly thinner than that in normal eyes (all $P<$ 0.001).

The macular VD and GCC thickness parameters are presented in Tables 1 and 2, respectively. The SSI in the normal group was significantly higher than that in the PPG $(69.3 \pm 5.9$ vs. $63.9 \pm 6.0, P=0.001)$ and early PG groups $(69.3 \pm 5.9$ vs. $65.1 \pm 5.6, P=0.013)$. Macular wiVD $(46.9 \% \pm 3.6 \%$ in PPG eyes; $43.1 \% \pm 5.3 \%$ in early PG eyes) and GCC thickness (92.4 \pm 7.9 in PPG eyes; $80.7 \pm 10.9$ in early PG eyes) were significantly lower in the PPG and early PG groups than in the control group $(50.9 \% \pm 2.8 \%$ for wiVD; $103.4 \pm 5.6$ for GCC thickness) (all $P<0.025$ in both univariate and multivariate analyses). In terms of regional measurements, the VD and GCC thickness parameters in PPG eyes were less than corresponding parameters in normal eyes in the multivariate analysis model with adjustment for age, gender, and SSI (all $P<0.025$ ), except for VD in the parafoveal region and in peri-temporal and peri-nasal sectors. In contrast, all the VD and GCC thickness parameters in the early PG group were significantly smaller compared to normal group in the multivariate analysis model (all $P<0.025)$. Examples of macular VD maps and GCC maps from a healthy eye (Fig. 1a), an eye with PPG (Fig. $1 b)$, and an eye with early PG (Fig. 1c) are shown.

The percent reduction of macular VD and GCC thickness in the PPG and early PG groups are displayed in Table 3. The percent reduction of wiVD was less than that of GCC thickness in early PG eyes $(15.4 \%$ vs. $22.0 \%$, $P=0.001$ ), while the percent reductions were comparable in PPG eyes ( $7.8 \%$ vs. $10.6 \%, P=0.117)$. In the PPG group, the percent reduction of VD was significantly less than that of GCC thickness in the perifoveal region (7.9\% vs. $12.2 \%, P=0.019$ ), while they were similar in the parafoveal region $(5.1 \%$ vs. $5.9 \%, P=0.338)$. In the early PG group, the percent reduction of VD was significantly less than that of GCC thickness in both the perifoveal $(16.1 \%$ vs. $22.9 \%, P=0.001)$ and parafoveal regions $(7.9 \%$ vs. $12.2 \%, P<0.05)$. Moreover, VD attenuation and GCC thinning were more severe in the perifoveal region compared to the parafoveal region in both groups (all $P<$ $0.05)$. The percent reduction of VD and GCC thickness in the early PG group were greater than that in the PPG group (all $P<0.05)$, except in the para-superior $(P=$ $0.126)$ and para-nasal sectors $(P=0.308)$.

The diagnostic ability of macular VD and GCC thickness in differentiating healthy controls from PPG or early PG eyes, as measured by the AUROC, is demonstrated in Table 4 and Fig. 2. AUROC values for the macular VD parameters were comparable to that of the corresponding GCC thickness parameters in both the 
Table 1 Macular VD in normal, pre-perimetric and early perimetric glaucoma eyes (Mean $\pm S D$ )

\begin{tabular}{llllll}
\hline & \multicolumn{2}{l}{ Macular VD (\%) } & & & \multicolumn{2}{c}{$P$ value (univariate, multivariate) } \\
\cline { 2 - 3 } & normal & PPG & $43.1 \pm 5.3$ & $<0.001,0.002$ & $b$ \\
\hline wi & $50.9 \pm 2.8$ & $46.9 \pm 3.6$ & $44.2 \pm 5.8$ & $<0.001,<0.001$ \\
wi SH & $51.0 \pm 2.9$ & $47.5 \pm 3.7$ & $41.9 \pm 5.7$ & $<0.001,<0.001$ & $<0.001,<0.001$ \\
wi IH & $50.9 \pm 2.8$ & $46.4 \pm 3.9$ & & & $<0.001,<0.001$ \\
Regional & & & $46.5 \pm 7.2$ & $0.022,0.333$ & $<0.001,0.004$ \\
Para & $52.4 \pm 3.3$ & $49.7 \pm 5.2$ & $46.0 \pm 7.9$ & $0.066,0.846$ & $<0.001,0.004$ \\
Para T & $52.4 \pm 3.3$ & $50.6 \pm 4.0$ & $48.3 \pm 6.4$ & $0.046,0.280$ & $0.001,0.019$ \\
Para S & $53.2 \pm 4.3$ & $50.5 \pm 6.0$ & $45.9 \pm 7.5$ & $0.003,0.052$ & $<0.001,0.002$ \\
Para N & $52.0 \pm 3.1$ & $47.7 \pm 6.8$ & $45.9 \pm 8.1$ & $0.171,0.851$ & $0.001,0.013$ \\
Para I & $51.9 \pm 4.0$ & $50.1 \pm 5.6$ & $43.6 \pm 5.6$ & $<0.001,0.002$ & $<0.001,<0.001$ \\
Peri & $51.9 \pm 2.8$ & $47.8 \pm 3.6$ & $40.2 \pm 4.5$ & $0.001,0.116$ & $<0.001,<0.001$ \\
Peri T & $46.9 \pm 3.4$ & $43.6 \pm 3.7$ & $44.5 \pm 6.6$ & $<0.001,0.009$ & $<0.001,<0.001$ \\
Peri S & $52.5 \pm 3.2$ & $48.1 \pm 3.9$ & $48.3 \pm 7.2$ & $0.001,0.026$ & $<0.001,<0.001$ \\
Peri N & $55.9 \pm 2.7$ & $53.1 \pm 3.5$ & $41.1 \pm 5.9$ & $<0.001,<0.001$ & $<0.001,<0.001$ \\
Peri I & $52.3 \pm 3.0$ & $46.5 \pm 4.6$ & & $<$
\end{tabular}

Univariate and multivariate analyses using a mixed effects model that was adjusted for age, sex, and SSI were used for comparison. The Bonferroni-corrected significance level (0.025) was used for multiple comparisons

VD Vessel density, PPG Pre-perimetric glaucoma, PG Perimetric glaucoma, wi Whole image, SH superior hemifield, IH Inferior hemifield, Para Parafoveal, Peri Perifoveal, $T$ Temporal, $S$ Superior, $N$ Nasal, I Inferior

a. Comparison between the PPG and normal group

b. Comparison between the early PG and normal group

PPG and early PG groups (all $P>0.05$ ). Globally, the AUROC values for wiVD and GCC thickness were 0.824 and 0.881 in the PPG group, respectively, and 0.918 and 0.977 in the early PG group, respectively. As to regional measurements, the perifoveal parameters had greater AUROC values than did the corresponding parafoveal parameters in the PPG group (both $P<0.05$ ). In the PG group, the AUROCs of GCC thickness in the perifoveal

Table 2 GCC thickness in normal, pre-perimetric and early perimetric glaucoma eyes (Mean \pm SD)

\begin{tabular}{|c|c|c|c|c|c|}
\hline & \multicolumn{3}{|c|}{ GCC thickness $(\mu \mathrm{m})$} & \multicolumn{2}{|c|}{$P$ value (univariate, multivariate) } \\
\hline & normal & PPG & early PG & $a$ & $b$ \\
\hline Global & $103.4 \pm 5.6$ & $92.4 \pm 7.9$ & $80.7 \pm 10.9$ & $<0.001,<0.001$ & $<0.001,<0.001$ \\
\hline Global SH & $103.2 \pm 5.6$ & $93.6 \pm 8.2$ & $84.2 \pm 12.3$ & $<0.001,<0.001$ & $<0.001,0.001$ \\
\hline Global IH & $103.6 \pm 6.0$ & $91.2 \pm 9.4$ & $77.1 \pm 12.4$ & $<0.001,<0.001$ & $<0.001,<0.001$ \\
\hline \multicolumn{6}{|l|}{ Regional } \\
\hline Para & $110.4 \pm 8.5$ & $103.9 \pm 9.7$ & $89.2 \pm 15.9$ & $0.010,<0.001$ & $<0.001,<0.001$ \\
\hline Para $\mathrm{T}$ & $102.5 \pm 7.9$ & $96.3 \pm 10.5$ & $81.3 \pm 13.5$ & $0.015,0.001$ & $<0.001,<0.001$ \\
\hline Para S & $113.2 \pm 9.1$ & $105.6 \pm 10.7$ & $93.4 \pm 16.0$ & $0.005,0.001$ & $<0.001,0.001$ \\
\hline Para N & $111.6 \pm 9.5$ & $107.1 \pm 8.0$ & $92.5 \pm 18.7$ & $0.058,<0.001$ & $<0.001,<0.001$ \\
\hline Para I & $114.3 \pm 8.6$ & $106.8 \pm 11.5$ & $89.5 \pm 20.3$ & $0.007,<0.001$ & $<0.001,<0.001$ \\
\hline Peri & $103.2 \pm 5.7$ & $90.6 \pm 8.0$ & $79.5 \pm 10.5$ & $<0.001,<0.001$ & $<0.001,<0.001$ \\
\hline Peri T & $87.2 \pm 4.2$ & $76.4 \pm 7.7$ & $68.2 \pm 7.9$ & $<0.001,<0.001$ & $<0.001,<0.001$ \\
\hline Peri S & $103.1 \pm 7.2$ & $90.0 \pm 9.8$ & $80.9 \pm 14.1$ & $<0.001,<0.001$ & $<0.001,0.002$ \\
\hline Peri N & $121.1 \pm 7.7$ & $111.2 \pm 8.8$ & $98.9 \pm 14.4$ & $<0.001,<0.001$ & $<0.001,0.001$ \\
\hline Peri I & $101.6 \pm 7.3$ & $84.9 \pm 11.7$ & $70.1 \pm 13.0$ & $<0.001,<0.001$ & $<0.001,<0.001$ \\
\hline
\end{tabular}

Univariate and multivariate analyses using mixed effects model with adjustment for age, gender, and SSI were used to for comparison. The Bonferroni-corrected significance level (0.025) was used for multiple comparisons

GCC Ganglion cell complex, PPG Pre-perimetric glaucoma, PG Perimetric glaucoma, SH Superior hemifield, IH Inferior hemifield, para Parafoveal, peri Perifoveal, $T$ Temporal, $S$ Superior, $N$ Nasal, I Inferior

a. Comparison between the PPG and normal group

b. Comparison between the early PG and normal group 

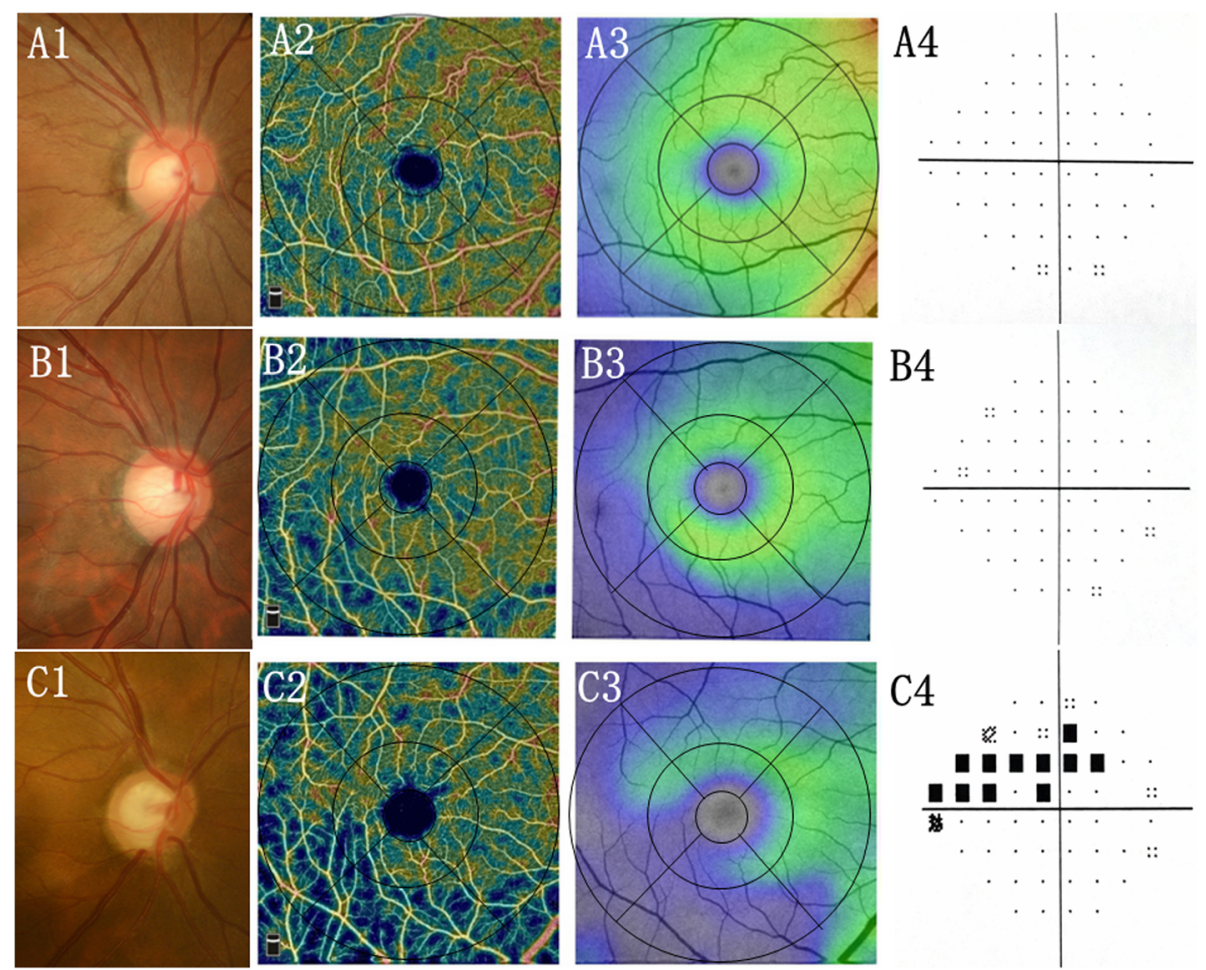

Fig. 1 Examples of eyes included in this study. A Normal-appearing optic nerve head with intact rim and RNFL (A1), intact GCC (A3), dense macular microvascular network (A2), and normal VF (A4) in normal eyes. Enlarged vertical cup-to-disc ratio with focal rim notching and RNFL defect (B1, C1), focal defects of macular microvascular network (B2, C2) matching the location of GCC loss (B3, C3) with normal VF (B4) in preperimetric glaucoma or with typical glaucomatous VF defects (C4) in early perimetric glaucoma. GCC, ganglion cell complex thickness. RNFL, peripapillary retinal nerve fiber layer; VF, visual field

and parafoveal regions were comparable $(P=0.261)$, while the perifoveal VD showed a significantly better $(P<0.05)$ AUROC than did the parafoveal VD.

The associations among wiVD, GCC thickness, pRNFL thickness, VF MD, and VF PSD are summarized in Additional file 2 and Additional file 3. Spearman correlation analysis showed that wiVD was negatively correlated with VF PSD and positively correlated with GCC thickness, pRNFL thickness, and VF MD in all glaucoma eyes. GCC thickness was negatively correlated with VF PSD and positively correlated with pRNFL thickness and VF MD (Additional file 2). Similar correlations were identified in mixed effects models that were adjusted for age, sex, and SSI (Additional file 3).

\section{Discussion}

In the human retina, RGCs are most densely located at the macula with approximately $50 \%$ of the being concentrated within $4.5 \mathrm{~mm}$ of the foveal center [33]. Thus, the changes of RGCs in terms of structure or microvasculature parameters in the macula may be sensitive indicators for glaucoma detection. In the current study, we evaluated macular VD and GCC thickness in healthy, PPG and early PG eyes. Our main findings were as follows: (1) A stepwise decrease of wiVD and GCC thickness was identified from normal eyes to PPG eyes to early PG eyes; (2) The diagnostic ability of macular $\mathrm{VD}$ is comparable to that of GCC thickness in detecting PPG or early PG; and (3) The percent reductions of VD and GCC thickness in the perifoveal region were greater than that in the parafoveal region in both groups.

Evaluations of the macular VD parameters in PPG eyes were not extensive $[8,26,29,30]$. Our study showed significant macular microvasculature dropout in PPG and early PG eyes, which was supported by Hou et al. [30] and Poli et al. [29]. In contrast, other studies did not show a significant decrease in macular VD $[8,26]$. The discrepancy may be partly attributed to the difference in glaucoma severity among the studies, as GCC atrophy was found in PPG eyes in our study, but remained unchanged in Triolo et al.'s [8] study. Scan sizes may also contribute to an inconsistency in results. Chen et al. [13] reported that no significant difference in the VD was found in the parafoveal area between glaucoma and healthy eyes, while the average VD value in the whole $6 \times 6 \mathrm{~mm}^{2}$ scanning field was lower in glaucoma eyes. Our regional analysis showed that the perifoveal region seemed more vulnerable to VD attenuation in PPG eyes; 
Table 3 Percent reduction of macular VD and GCC thickness in pre-perimetric and early perimetric glaucoma eyes (mean, 95\% confidence interval)

\begin{tabular}{|c|c|c|c|c|c|c|}
\hline & Pre-perimetric $g$ & & & Early perimetric & & \\
\hline & VD (\%) & GCC Thickness (\%) & $P$ & VD (\%) & GCC Thickness (\%) & $P$ \\
\hline Global *** & $7.8(5.0,10.7)$ & $10.6(7.5,13.7)$ & 0.117 & $15.4(10.6,20.0)$ & $22.0(17.3,26.6)$ & 0.001 \\
\hline Global SH **** & $6.9(4.0,9.8)$ & $9.3(6.1,12.5)$ & 0.096 & $13.3(8.2,18.4)$ & $18.4(13.1,23.6)$ & 0.004 \\
\hline Global IH *** & $8.9(5.7,12.0)$ & $11.7(8.0,15.4)$ & 0.152 & $17.6(12.7,22.6)$ & $25.4(20.1,30.7)$ & 0.001 \\
\hline Regional & & & & & & \\
\hline Para $* * *$ & $5.1(1.1,9.0)$ & $5.9(2.3,9.4)$ & 0.338 & $11.2(5.2,17.3)$ & $19.2(12.9,25.6)$ & 0.001 \\
\hline Peri $* * *$ & $7.9(5.1,10.7)$ & $12.2(9.1,15.3)$ & 0.019 & $16.1(11.3,20.8)$ & $22.9(18.4,27.4)$ & 0.001 \\
\hline & $P=0.020$ & $P<0.001$ & & $P=0.003$ & $P=0.040$ & \\
\hline Para $T^{* * * *}$ & $3.5(0.4,6.5)$ & $6.0(1.9,10.2)$ & 0.128 & $12.2(5.6,18.9)$ & $20.7(14.9,26.6)$ & 0.001 \\
\hline Para $S^{* *}$ & $5.2(0.6,9.7)$ & $6.7(2.9,10.5)$ & 0.234 & $9.2(3.9,14.6)$ & $17.5(11.2,23.7)$ & 0.001 \\
\hline Para N ** & $8.2(3.0,13.4)$ & $4.0(1.2,7.0)$ & 0.873 & $11.8(5.4,18.2)$ & $17.1(9.7,24.6)$ & 0.018 \\
\hline Para I*** & $3.4(-0.01,7.7)$ & $6.6(2.5,10.6)$ & 0.206 & $11.5(4.6,18.4)$ & $21.7(13.8,29.6)$ & 0.003 \\
\hline Peri $\top^{*} * * *$ & $7.1(4.0,10.3)$ & $12.4(8.8,16.0)$ & 0.014 & $14.2(9.9,18.5)$ & $21.8(17.8,25.8)$ & 0.001 \\
\hline Peri $S^{*} * *$ & $8.4(5.4,11.5)$ & $12.8(9.0,16.7)$ & 0.026 & $15.1(9.6,20.7)$ & $21.5(15.4,27.6)$ & 0.005 \\
\hline Peri $N * * *$ & $5.0(2.5,7.5)$ & $8.1(5.2,11.1)$ & 0.103 & $13.6(7.8,19.3)$ & $18.3(13.0,23.6)$ & 0.013 \\
\hline Peri I*** & $11.0(7.5,14.5)$ & $16.5(11.8,21.5)$ & 0.023 & $21.3(16.3,26.3)$ & $31.0(25.3,36.7)$ & 0.001 \\
\hline
\end{tabular}

A clustered Wilcoxon signed rank test using the Rosner-Glynn-Lee method was used to compare the percent reduction of macular VD or GCC thickness between different areas within each individual group

$P, P$ values between macular VD and GCC thickness or between the different regions in the individual group

VD Vessel density, GCC Ganglion cell complex, SH Superior hemifield, IH Inferior hemifield, para Parafoveal, peri Perifoveal, $T$ Temporal, $S$ superior, $N$ Nasal, I Inferior Multivariate analysis using a mixed effects model that was adjusted for age, sex, and SSI was used for the comparison between the PPG and early PG groups in terms of the percent reduction of VD $(*, P<0.05)$ and GCC thickness $(* *, P<0.05)$

thus, the larger scan size used in our study appears more likely to cover the affected area.

Previous studies have reported that the AUROC values for macular VD that ranged from 0.562 to 0.800 for distinguishing between early glaucoma and normal eyes $[26,27,30,34]$. However, little is known about the diagnostic ability of macular VD in detecting PPG. In our study, wiVD had good diagnostic ability in detecting PPG and early PG eyes (AUROC: 0.824 and 0.918, respectively), which was better than that presented in a previous study [30]. The improved diagnostic ability may be due to the advantage of the larger scan size that we used, as previously mentioned. In addition, macular measurements may offer some advantages, such as less inter-individual structural variability and insusceptibility to the impacts of disc tilting and large peripapillary atrophy [35]. Thus, macular VD measurement may be a supplementary tool for identifying early glaucomatous damage. The AUROCs of wiVD and GCC thickness were comparable in our study, which is consistent with Hou et al.'s results [30]. However, other studies have reported that macular VD performed worse than GCC thickness in differentiating between glaucoma from healthy eyes $[9,13,15,36]$. The inconsistency may be attributed to the fact that the glaucoma severities differed from our study or the fact that the regions of interest for macular VD measurement and GCC thickness were not the same.

Table 4 Diagnostic ability of macular vessel density and GCC thickness parameters in differentiating pre-perimetric /early perimetric glaucoma eyes from normal eyes (mean, 95\% confidence interval)

\begin{tabular}{|c|c|c|c|c|c|c|}
\hline & \multicolumn{3}{|c|}{ Normal vs. pre-perimetric glaucoma } & \multicolumn{3}{|c|}{ Normal vs. early perimetric glaucoma } \\
\hline & VD & GCC Thickness & $p^{a}$ & VD & GCC Thickness & $p^{a}$ \\
\hline wi & $0.824(0.701,0.912)$ & $0.881(0.768,0.952)$ & 0.419 & $0.918(0.809,0.976)$ & $0.977(0.892,0.999)$ & 0.109 \\
\hline para & $0.635(0.497,0.758)$ & $0.694(0.557,0.809)$ & 0.580 & $0.780(0.645,0.882)$ & $0.902(0.788,0.966)$ & 0.094 \\
\hline peri & $0.819(0.694,0.908)$ & $0.905(0.798,0.967)$ & 0.157 & $0.918(0.809,0.976)$ & $0.959(0.865,0.994)$ & 0.286 \\
\hline$P^{b}$ & $<0.001$ & $<0.001$ & & 0.005 & 0.261 & \\
\hline
\end{tabular}

VD Vessel density, GCC Ganglion cell complex, wi Whole image, para Parafoveal, peri Perifoveal

${ }^{a}$ Comparison between the AUROCs for macular VD and GCC thickness in the individual group using Delong's method

${ }^{b}$ Comparison of the AUROCs for each individual parameter between the parafoveal and perifoveal regions using Delong's method 

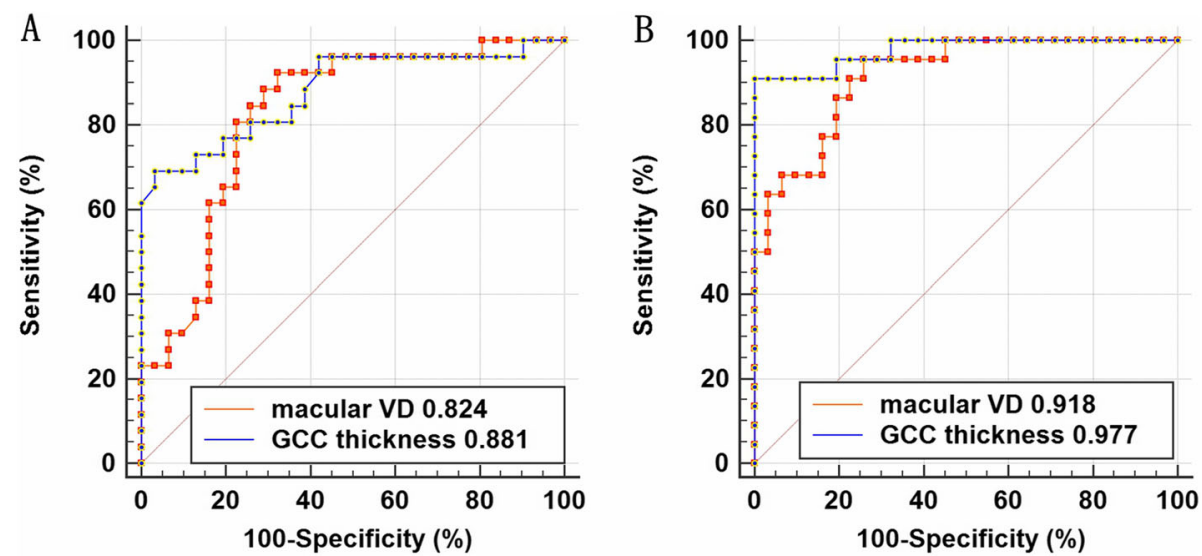

Fig. 2 AUROC values for macular VD and GCC thickness. Macular VD showed a lower AUROC value than did GCC thickness in the pre-perimetric glaucoma (A) and early perimetric glaucoma groups (B), but the differences were not statistically significant (both $P>0.05$ ). AUROC, area under the receiver operating characteristic curve; VD, vessel density; GCC, ganglion cell complex

Glaucoma usually starts as focal lesions [2]. The regional mapping of the OCT/OCTA measurements may help us understand early glaucomatous changes. Lin et al. [37] reported that the macular RNFL and macular ganglion cell layer thickness in PPG eyes decreased more in the perifoveal region than in the parafoveal region. However, to the best of our knowledge, there is no study comparing vessel densities in the perifoveal region and parafoveal region in PPG eyes. In this study, we found that VD attenuation and GCC thinning were greater in the perifoveal region in both groups. The peri-inferior sector appeared most vulnerable to glaucomatous damage, which was concordant with the findings of Hood et al. $[18,38]$. In addition, we found that the percent reduction of GCC thickness was greater than that of macular VD in eyes with PPG, an earlier course than what reported by Hou et al. [30]. One possible explanation for the mismatch between neural atrophy and vascular insufficiency is that microvascular dropouts can be secondary to neurodegeneration, such as GCC thinning. However, given the sensitivities of the imaging device to detect various parameters may be different, we cannot completely exclude the possibility that a less prominent and undetectable hemodynamic deficiency may occur prior to RGC degeneration. Additional longitudinal studies are required to investigate this issue.

The strengths of the current study included the following: First, macular VD and GCC thickness were acquired over the same scan size, which allowed a fair comparison between the two measurements for detection of glaucoma. Second, we used the AngioRetina HD $6 \times 6 \mathrm{~mm}^{2}$ mode with the ETDRS segmentation algorithm, which allowed us to evaluate focal changes in the early course of glaucoma. However, there were some limitations to our study. First, the sample size was small. Second, it is impossible to clarify the temporal sequence of macular
VD attenuation and GCC thinning due to the crosssectional design. Third, although the subjects were younger in our study, age has been shown not to affect macular VD measurements [39]. However, we cannot completely rule out the impact of other potential confounding factors, such as systemic disease or hypotensive eye drops, on the VD measurements.

\section{Conclusions}

The current study demonstrated that wiVD and GCC thickness significantly decreased in PPG and early PG eyes compared to controls. The perifoveal region appeared more vulnerable to early glaucomatous damage. The diagnostic accuracy of macular VD was comparable to that of GCC thickness in distinguishing eyes with PPG or early PG from healthy eyes. Overall, macular VD measurements with the larger scan size may show promise to detect early glaucoma, and the regional mapping may be helpful for understanding early glaucomatous damage.

\section{Supplementary information}

Supplementary information accompanies this paper at https://doi.org/10 1186/s12886-020-1304-x

Additional file 1. The demographic and ocular characteristics of the subjects.

Additional file 2. Spearman correlation coefficient matrix on wiVD, GCC thickness, pRNFL thickness, VF MD, and VF PSD.

Additional file 3. Mixed-effect model analysis of macular VD and GCC thickness.

Abbreviations

AL: Axial length; AUROC: Area under the receiver operating characteristic curve; BP: Blood pressure; CCT: Central corneal thickness; IOP: Intraocular pressure; MD: Mean deviation; MOPP: Mean ocular perfusion pressure; OCT: Optical coherence tomography; OCTA: Optical coherence tomography angiography; PG: Periperimetric glaucoma; POAG: Primary open-angle 
glaucoma; PPG: Pre-perimetric glaucoma; PSD: Pattern standard deviation; RGC: Retinal ganglion cell; RNFL: Retinal nerve fiber layer; SSI: Signal strength index; VD: Vessel density; VF: Visual field; wiVD: Whole image vessel density

\section{Acknowledgements}

None.

\section{Authors' contributions}

NW, HW and YW made contributions to conception and design; NW and HW were responsible for provision of materials and patients; YW, HW contributed to the acquisition of data; YW, HW, KC, ML, CX participated in data analysis and interpretation; YW, DLS, CX were involved in drafting the manuscript; NW contributed as corresponding author. All authors approved the final manuscript.

\section{Funding}

None.

\section{Availability of data and materials}

All data included in this study are available from the corresponding author on reasonable request.

\section{Ethics approval and consent to participate}

The study was approved by the Institutional Review Board of Beijing Tongren Hospital and followed the tenets of the Declaration of Helsinki. Written informed consent was obtained from all subjects.

\section{Consent for publication}

Not available.

\section{Competing interests}

The authors declare that they have no competing interests.

\section{Author details}

${ }^{1}$ Beijing Institute of Ophthalmology, Beijing Tongren Eye Centre, Beijing Tongren Hospital, Capital Medical University, 1 Dongjiaominxiang Street, Dongcheng District, Beijing 100730, China. ${ }^{2}$ Beijing Ophthalmology \& Visual Sciences Key Laboratory, 1 Dongjiaominxiang Street, Dongcheng District, Beijing 100730, China. ${ }^{3}$ Department of Ophthalmology, Boston University School of Medicine, Boston, MA 02118, USA. ${ }^{4}$ Department of Anatomy and Neurobiology, Boston University School of Medicine, Boston, MA 02118, USA. ${ }^{5}$ Department of Ophthalmology, Beijing Tongren Eye Centre, Beijing Tongren Hospital, Capital Medical University, Beijing 100730, China.

Received: 5 September 2019 Accepted: 31 December 2019

Published online: 08 January 2020

\section{References}

1. Tham YC, Li X, Wong TY, Quigley HA, Aung T, Cheng CY. Global prevalence of glaucoma and projections of glaucoma burden through 2040: a systematic review and meta-analysis. Ophthalmology. 2014;121(11):2081-90.

2. Weinreb RN, Aung T, Medeiros FA. The pathophysiology and treatment of glaucoma: a review. Jama. 2014;311(18):1901-11

3. Hwang JC, Konduru R, Zhang X, Tan O, Francis BA, Varma R, et al. Relationship among visual field, blood flow, and neural structure measurements in glaucoma. Invest Ophthalmol Vis Sci. 2012;53(6):3020-6.

4. Flammer J, Orgul S, Costa VP, Orzalesi N, Krieglstein GK, Serra LM, et al. The impact of ocular blood flow in glaucoma. Prog Retin Eye Res. 2002;21(4): 359-93.

5. Hayreh SS. Blood flow in the optic nerve head and factors that may influence it. Prog Retin Eye Res. 2001;20(5):595-624.

6. Cherecheanu AP, Garhofer G, Schmidl D, Werkmeister R, Schmetterer L. Ocular perfusion pressure and ocular blood flow in glaucoma. Curr Opin Pharmacol. 2013:13(1):36-42.

7. Jia Y, Wei E, Wang $X$, Zhang $X$, Morrison JC, Parikh M, et al. Optical coherence tomography angiography of optic disc perfusion in Glaucoma. Ophthalmology. 2014;121(7):1322-32.

8. Triolo G, Rabiolo A, Shemonski ND, Fard A, Di Matteo F, Sacconi R, et al. Optical coherence tomography angiography macular and Peripapillary vessel perfusion density in healthy subjects, Glaucoma suspects, and Glaucoma patients. Invest Ophthalmol Vis Sci. 2017;58(13):5713-22.
9. Penteado RC, Zangwill LM, Daga FB, Saunders LJ, Manalastas PIC, Shoji T, et al. Optical coherence tomography angiography macular vascular density measurements and the central 10-2 visual field in Glaucoma. J Glaucoma. 2018;27(6):481-9.

10. Kromer R, Glusa P, Framme C, Pielen A, Junker B. Optical coherence tomography angiography analysis of macular flow density in glaucoma. Acta Ophthalmol. 2019;97(2):e199-206.

11. Moghimi S, Zangwill LM, Penteado RC, Hasenstab K, Ghahari E, Hou H, et al. Macular and optic nerve head vessel density and progressive retinal nerve Fiber layer loss in Glaucoma. Ophthalmology. 2018;125(11):1720-8.

12. Richter GM, Madi I, Chu Z, Burkemper B, Chang R, Zaman A, et al. Structural and functional associations of macular microcirculation in the ganglion cellinner Plexiform layer in Glaucoma using optical coherence tomography angiography. J Glaucoma. 2018;27(3):281-90.

13. Chen HS, Liu CH, Wu WC, Tseng HJ, Lee YS. Optical coherence tomography angiography of the superficial microvasculature in the macular and Peripapillary areas in glaucomatous and healthy eyes. Invest Ophthalmol Vis Sci. 2017;58(9):3637-45.

14. Shoji T, Zangwill LM, Akagi T, Saunders LJ, Yarmohammadi A, Manalastas $\mathrm{PIC}$, et al. Progressive macula vessel density loss in primary open-angle Glaucoma: a longitudinal study. Am J Ophthalmol. 2017;182:107-17.

15. Takusagawa HL, Liu L, Ma KN, Jia Y, Gao SS, Zhang M, et al. Projectionresolved optical coherence tomography angiography of macular retinal circulation in Glaucoma. Ophthalmology. 2017;124(11):1589-99.

16. Yarmohammadi A, Zangwill LM, Diniz-Filho A, Saunders LJ, Suh MH, Wu Z, et al. Peripapillary and macular vessel density in patients with Glaucoma and single-Hemifield visual field defect. Ophthalmology. 2017;124(5):709-19.

17. Hood DC, Raza AS, de Moraes CG, Liebmann JM, Ritch R. Glaucomatous damage of the macula. Prog Retin Eye Res. 2013;32:1-21.

18. Hood DC. Improving our understanding, and detection, of glaucomatous damage: an approach based upon optical coherence tomography (OCT). Prog Retin Eye Res. 2017:57:46-75.

19. Quigley HA, Dunkelberger GR, Green WR. Retinal ganglion cell atrophy correlated with automated perimetry in human eyes with glaucoma. Am J Ophthalmol. 1989;107(5):453-64.

20. Kerrigan-Baumrind LA, Quigley HA, Pease ME, Kerrigan DF, Mitchell RS Number of ganglion cells in Glaucoma eyes compared with threshold visual field tests in the same persons. Invest Ophthalmol Vis Sci. 2000;41(3):741-8.

21. Lisboa R, Paranhos A Jr, Weinreb RN, Zangwill LM, Leite MT, Medeiros FA. Comparison of different spectral domain OCT scanning protocols for diagnosing preperimetric glaucoma. Invest Ophthalmol Vis Sci. 2013;54(5): 3417-25.

22. Rolle T, Briamonte C, Curto D, Grignolo FM. Ganglion cell complex and retinal nerve fiber layer measured by fourier-domain optical coherence tomography for early detection of structural damage in patients with preperimetric glaucoma. Clin Ophthalmol. 2011;5:961-9.

23. Cennamo G, Montorio D, Romano MR, Cardone DM, Minervino C, Reibaldi $M$, et al. Structure-functional parameters in differentiating between patients with different degrees of Glaucoma. J Glaucoma. 2016;25(10):e884-e8.

24. Kim MJ, Jeoung JW, Park KH, Choi YJ, Kim DM. Topographic profiles of retinal nerve fiber layer defects affect the diagnostic performance of macular scans in preperimetric glaucoma. Invest Ophthalmol Vis Sci. 2014; 55(4):2079-87.

25. Chen MJ, Yang HY, Chang YF, Hsu CC, Ko YC, Liu CJ. Diagnostic ability of macular ganglion cell asymmetry in Preperimetric Glaucoma. BMC Ophthalmol. 2019;19(1):12.

26. Chung JK, Hwang YH, Wi JM, Kim M, Jung JJ. Glaucoma diagnostic ability of the optical coherence tomography angiography vessel density parameters. Curr Eye Res. 2017;42(11):1458-67.

27. Kurysheva NI, Maslova EV, Zolnikova IV, Fomin AV, Lagutin MB. A comparative study of structural, functional and circulatory parameters in glaucoma diagnostics. PLoS One. 2018;13(8):e0201599.

28. Rolle T, Dallorto L, Tavassoli M, Nuzzi R. Diagnostic ability and discriminant values of OCT-angiography parameters in early Glaucoma diagnosis. Ophthalmic Res. 2019;61(3):143-52.

29. Poli M, Cornut PL, Nguyen AM, De Bats F, Denis P. Accuracy of peripapillary versus macular vessel density in diagnosis of early to advanced primary open angle glaucoma. J Fr Ophtalmol. 2018;41(7):619-29.

30. Hou H, Moghimi S, Zangwill LM, Shoji T, Ghahari E, Penteado RC, et al. Macula vessel density and thickness in early primary open-angle Glaucoma. Am J Ophthalmol. 2019;199:120-32. 
31. Yarmohammadi A, Zangwill LM, Diniz-Filho A, Suh MH, Manalastas PI, Fatehee $\mathrm{N}$, et al. Optical coherence tomography angiography vessel density in healthy, Glaucoma suspect, and Glaucoma eyes. Invest Ophthalmol Vis Sci. 2016;57(9):OCT451-OCT9.

32. Jia Y, Morrison JC, Tokayer J, Tan O, Lombardi L, Baumann B, et al. Quantitative OCT angiography of optic nerve head blood flow. Biomedical Optics Express. 2012;3(12):3127-37.

33. Curcio CA, Allen KA. Topography of ganglion cells in human retina. J Comp Neurol. 1990;300(1):5-25.

34. Yarmohammadi A, Zangwill LM, Manalastas PIC, Fuller NJ, Diniz-Filho A, Saunders $L$, et al. Peripapillary and macular vessel density in patients with primary open-angle Glaucoma and unilateral visual field loss. Ophthalmology. 2018;125(4):578-87.

35. Hirasawa H, Araie M, Tomidokoro A, Saito H, Iwase A, Ohkubo S, et al. Reproducibility of thickness measurements of macular inner retinal layers using SD-OCT with or without correction of ocular rotation. Invest Ophthalmol Vis Sci. 2013;54(4):2562-70.

36. Wan KH, Lam AKN, Leung CK. Optical coherence tomography angiography compared with optical coherence tomography macular measurements for detection of Glaucoma. JAMA Ophthalmol. 2018;136(8):866-74.

37. Lin JP, Lin PW, Lai IC, Tsai JC. Segmental inner macular layer analysis with spectral-domain optical coherence tomography for early detection of normal tension glaucoma. PLoS One. 2019;14(1):e0210215.

38. Hood DC, Raza AS, de Moraes CG, Johnson CA, Liebmann JM, Ritch R. The nature of macular damage in Glaucoma as revealed by averaging optical coherence tomography data. Transl Vis Sci Technol. 2012:1(1):3.

39. Rao HL, Pradhan ZS, Weinreb RN, Reddy HB, Riyazuddin M, Sachdeva S, et al. Determinants of Peripapillary and macular vessel densities measured by optical coherence tomography angiography in Normal eyes. I Glaucoma. 2017:26(5):491-7.

\section{Publisher's Note}

Springer Nature remains neutral with regard to jurisdictional claims in published maps and institutional affiliations.

Ready to submit your research? Choose BMC and benefit from:

- fast, convenient online submission

- thorough peer review by experienced researchers in your field

- rapid publication on acceptance

- support for research data, including large and complex data types

- gold Open Access which fosters wider collaboration and increased citations

- maximum visibility for your research: over $100 \mathrm{M}$ website views per year

At $\mathrm{BMC}$, research is always in progress.

Learn more biomedcentral.com/submissions 